\title{
Lung Transplantation in a Patient with Pre-transplant Colonization of Extensively Drug-resistant Acinetobacter baumannii
}

\author{
Hwa Young Lee, M.D., Hea Yon Lee, M.D., Sae Bom Shin, M.D., Kab Soo Shin, M.D., Bong Woo Lee, M.D., \\ Hwan Wook Kim, M.D., Ph.D.*, Seok Lee, M.D., Ph.D. ${ }^{\dagger}$, and Seok Chan Kim, M.D., Ph.D. \\ Division of Respiratory, Allergy and Critical Care, Department of Internal Medicine; *Department of Thoracic and Cardiovascular Surgery; 'Division of Hematology and Oncology, \\ Catholic Blood and Marrow Transplantation Center, Department of Internal Medicine, St. Mary's Hospital, The Catholic University of Korea, Seoul, Korea
}

\begin{abstract}
Colonization of the pre-transplant lung by multidrug-resistant bacteria affects short- and long-term outcomes of lung transplantation. However, there are no case reports on the colonization of a pre-transplant lung by drug-resistant Acinetobacter baumannii. We report a case of extensively drug resistant (XDR) A. baumannii colonization in the tracheobronchial tree that caused severe infectious complications after bilateral lung transplantation. A 23-year-old man diagnosed with bronchiolitis obliterans syndrome (BOS) 4 years earlier with a history of allogenic bone marrow transplantation for acute lymphoblastic leukemia was admitted to the hospital with dyspnea. Due to progressive hypercapnic respiratory failure, long-term mechanical ventilation was started after a tracheostomy was performed, and the patient underwent a bilateral lung transplantation to treat end-stage BOS. After the transplantation, the colonization of XDR A. baumannii caused severe bacterial pneumonia in the early postoperative period. Combined treatment with colistin and meropenem led to recovery from the pneumonia but caused drug-induced renal failure. Because many centers are willing to transplant candidates who are on mechanical ventilation or extracorporeal life support, the incidence of XDR A. baumannii colonization of pretransplant lungs is expected to increase. Further studies are needed to examine pre-transplant management strategies in patients colonized with XDR A. baumannii.
\end{abstract}

Key Words: Acinetobacter baumannii; drug resistance; lung transplantation.

In recent decades, the indications for lung transplantation (LT) have been extended, and favorable outcomes in patients diagnosed with chronic graft-versus-host disease-associated bronchiolitis obliterans syndrome (BOS) after bone marrow transplantation (BMT) have been published.[1-3] With the progress in immunosuppressant modulation and antibiotics, successful LT has been performed in patients colonized with drug-resistant pathogens, although colonization with highly resistant bacteria is generally considered to be a relative contraindication to LT.[4] Extensively drug-resistant (XDR) Acinetobacter baumannii is a nosocomial pathogen frequently associated with prolonged ventilation in the intensive care unit or the long-term use of broadspectrum antibiotics.[5] In recent years, acquired infections of the transplanted lung with multidrug-resistant $A$. baumannii in the postoperative period have had devastating outcomes.[6,7] In the pretransplant setting, however, there are no reports describing the impact of colonization by multidrug-resistant $A$.

Received on December 24, 2014 Revised on March 26, 2015 Accepted on April 20, 2015

Correspondence to: Seok Chan Kim, Division of Respiratory, Allergy and Critical Care Medicine, Department of Internal Medicine, College of Medicine, Catholic University of Korea, 222 Banpo-Daero, Seocho-Gu, Seoul 137-701, Korea Tel: +82-2-2258-6062, Fax: +82-2-2258-3589 E-mail: cmcksc@catholic.ac.kr

${ }^{*}$ No potential conflict of interest relevant to this article was reported. baumannii on the post-transplant outcomes.[8] We herein report a case of pretransplant colonization of XDR A. baumannii in a patient who underwent bilateral lung transplantation for BOS after stem cell transplantation that was complicated by a severe infection.

(c) This is an Open Access article distributed under the terms of the Creative Commons Attribution Non-Commercial License (http://creativecommons.org/ licenses/by-nc/3.0/) which permits unrestricted non-commercial use, distribution, and reproduction in any medium, provided the original work is properly cited. 


\section{Case Report}

A 23-year-old man was admitted to the hospital with dyspnea. He had been diagnosed with BOS in 2010 after alloBMT for acute lymphoblastic leukemia. The hematological malignancy had shown a complete response since 2009. The patient developed recurrent pneumothorax in 2012 and 2014 and recovered after chest tube drainage. Pulmonary function tests performed in 2012 showed evidence of BOS with severe obstructive lung disease, a reduced ratio of the forced expiratory volume in one second $\left(\mathrm{FEV}_{1}\right)$ to forced vital capacity ( $50 \%$ of expected), $\mathrm{FEV}_{1}(0.62 \mathrm{~L}, 15 \%$ of expected), diffusion capacity ( $33 \%$ of predicted), and increased residual volume (3.26 L, $238 \%$ of expected). He had been taking an inhaled corticosteroid to treat the BOS and low-dose mycophenolate mofetil (MMF) for extensive chronic oral, eye, and lung graft-versus-host disease for 5 years. Before admission, he had been treated for a nontuberculous Mycobacterium intracellulare lung infection with rifampin, ethambutol, and clarithromycin for 3 months with sputum converting to negative after 1 month of treatment. At the time of admission, the patient had a cough and whitish sputum, but did not have a fever; his lungs were clear with no wheezing on auscultation. The white blood cell count was $4,770 / \mathrm{mm}^{3}$

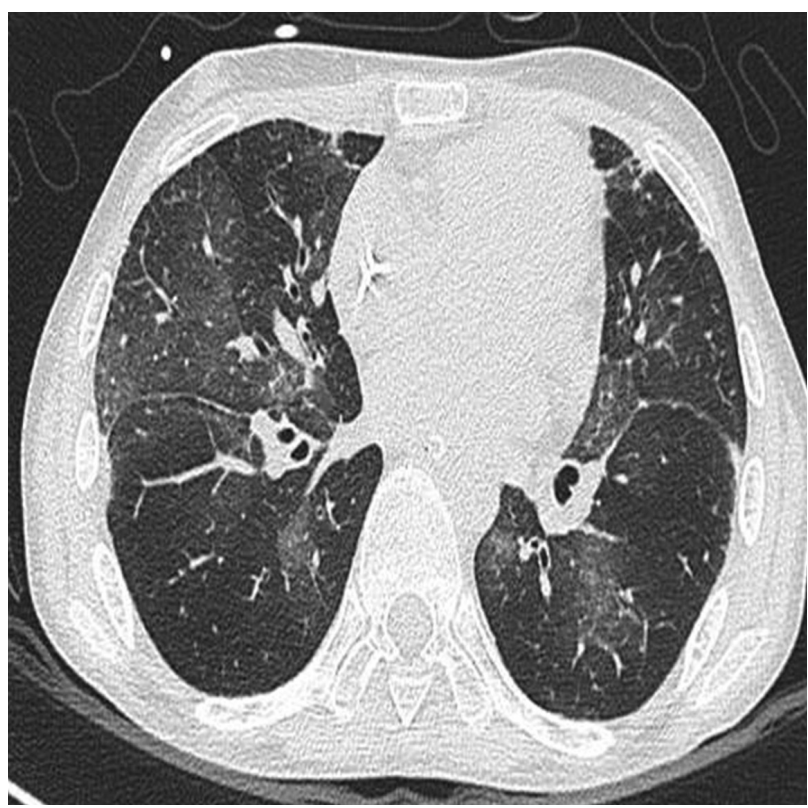

Fig. 1. Chest computed tomography taken before lung transplantation showed heterogeneously decreased lung attenuation with bronchial dilatation, suggesting advanced BOS. with $62 \%$ segmented neutrophils; the high-sensitivity Creactive protein (hs-CRP) level was $2.67 \mathrm{mg} / \mathrm{dL}$ (reference range, $<0.47 \mathrm{mg} / \mathrm{dL}$ ). Chest computed tomography showed heterogeneously decreased lung attenuation with bronchial dilatation, suggesting advanced BOS (Fig. 1) without evidence of pneumothorax or progressive acute infection. An initial arterial blood gas examination showed a $\mathrm{pH}$ of 7.381, $\mathrm{PaCO}_{2}$ of $72.5 \mathrm{mmHg}, \mathrm{PaO}_{2}$ of $48.7 \mathrm{mmHg}, \mathrm{HCO}_{3}$ of $>40$ $\mathrm{mmol} / \mathrm{L}$, and $\mathrm{SPO}_{2}$ of $93.8 \%$ on $2 \mathrm{~L} / \mathrm{min}$ oxygen support via nasal prongs; the $\mathrm{PaCO}_{2}$ was higher than it had been 3 months previously. Four days after admission, an endotracheal tube was inserted and mechanical ventilation was started due to progressive hypercapnia. After intubation, the arterial blood gas examination showed hypercapnic respiratory acidosis on ventilatory support $\left(\mathrm{pH}, 7.192 ; \mathrm{PaCO}_{2}, 116\right.$ $\mathrm{mmHg} ; \mathrm{PaO}_{2}, 401 \mathrm{mmHg} ; \mathrm{HCO}_{3}, 40 \mathrm{mmol} / \mathrm{L}$; and $\mathrm{SpO}_{2}$, $99 \%$ ). With ventilatory support and inhaled corticosteroids, the acute hypercapnic respiratory failure was partly relieved. However, ventilator weaning 9 days after admission failed, and a tracheostomy was performed for long-term mechanical ventilation. XDR A. baumannii had been isolated from the sputum and bronchial washing fluid cultures 10 times since the fourth day of admission. All isolates were resistant to all cephalosporins, quinolones, aminoglycosides, and

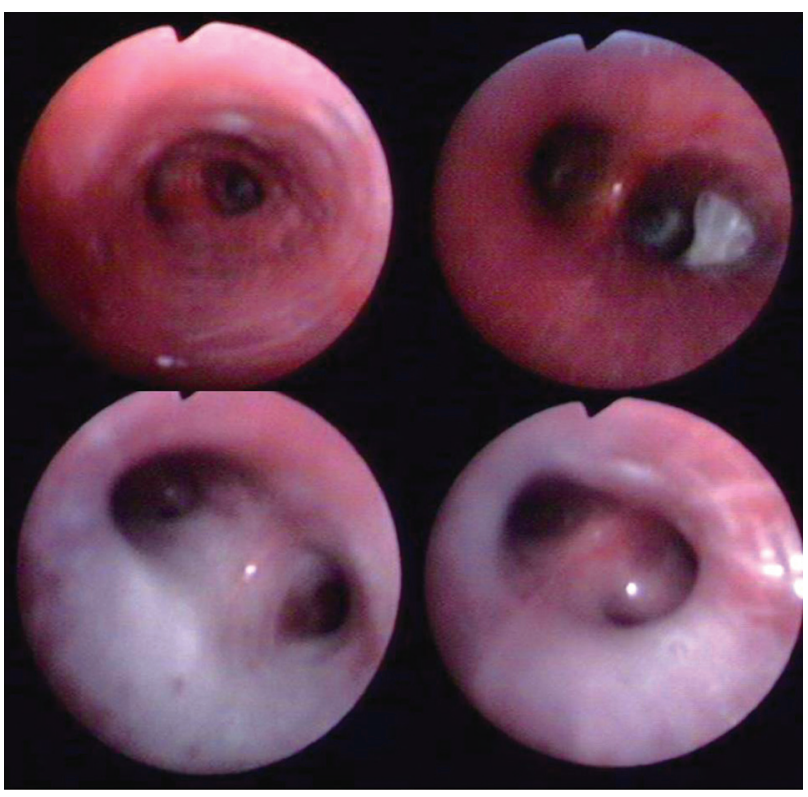

Fig. 2. Bronchoscopy performed on POD 7 showed erythematous, inflammatory mucosa with profuse purulent sputum in both bronchi. 
carbapenems, but susceptible to colistin (minimum inhibitory concentration $[\mathrm{MIC}], \leq 0.5)$ and tigecycline $(\mathrm{MIC}=$ $2)$. Because there were no signs or symptoms of systemic inflammation, the pathogen was considered to be colonizing and targeted therapy was not planned. To treat the endstage BOS, the patient was listed for lung transplantation. After mechanical ventilation for 51 days, he underwent bilateral LT from a cadaveric unrelated donor. The postoperative antimicrobial prophylaxis regimen comprised colistin, teicoplanin, trimethoprim-sulfamethoxazole, ganciclovir, and itraconazole on the day of the operation, with immunosuppression using basiliximab, tacrolimus (trough level 10-15 $\mu \mathrm{g} / \mathrm{mL}), \mathrm{MMF}$, and corticosteroids. Bronchoscopy on postoperative day (POD) 7 showed that the anastomosis was intact, but the mucosa of the transplanted lung showed erythematous inflammatory changes with profuse, thick purulent sputum in both bronchi (Fig. 2). On POD 13, a fever of $37.7^{\circ} \mathrm{C}$ occurred with hypotension, an elevated serum hs-CRP level $(20.95 \mathrm{mg} / \mathrm{dL})$, and leukocytosis $(20.33 \times$ $\left.10^{9} / \mathrm{L}\right)$. A chest X-ray showed extensive infiltration of both
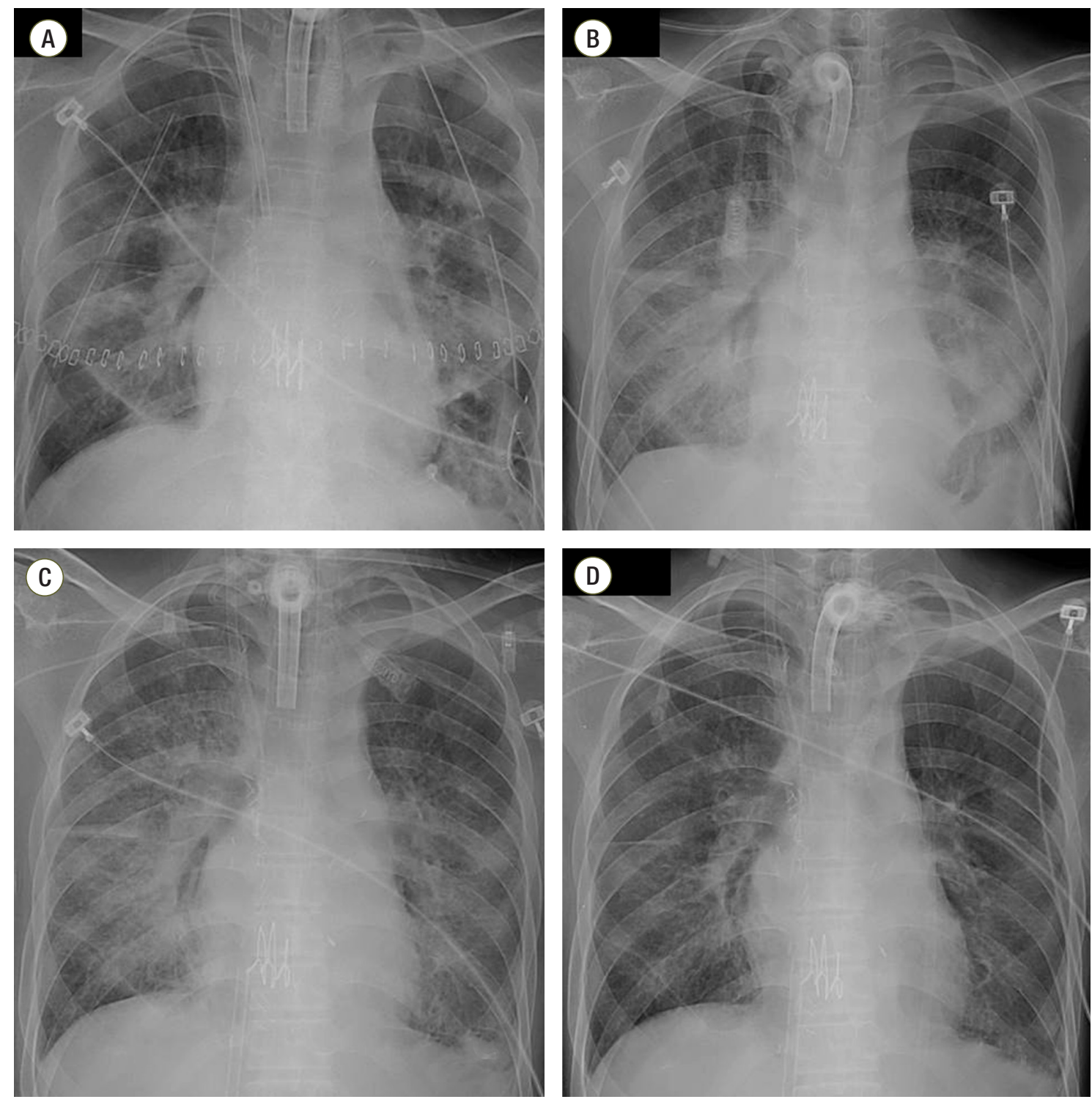

Fig. 3. Chest x-ray taken on POD 1 (A), POD 13 (B), POD 18 (C), and POD 27 (D), respectively. On POD 13, extensive bilateral infiltrations developed along with hypoxemic respiratory failure, which improved on POD 18. Chest x-ray on POD 27 showed almost complete regression of the pneumonic infiltrations. 


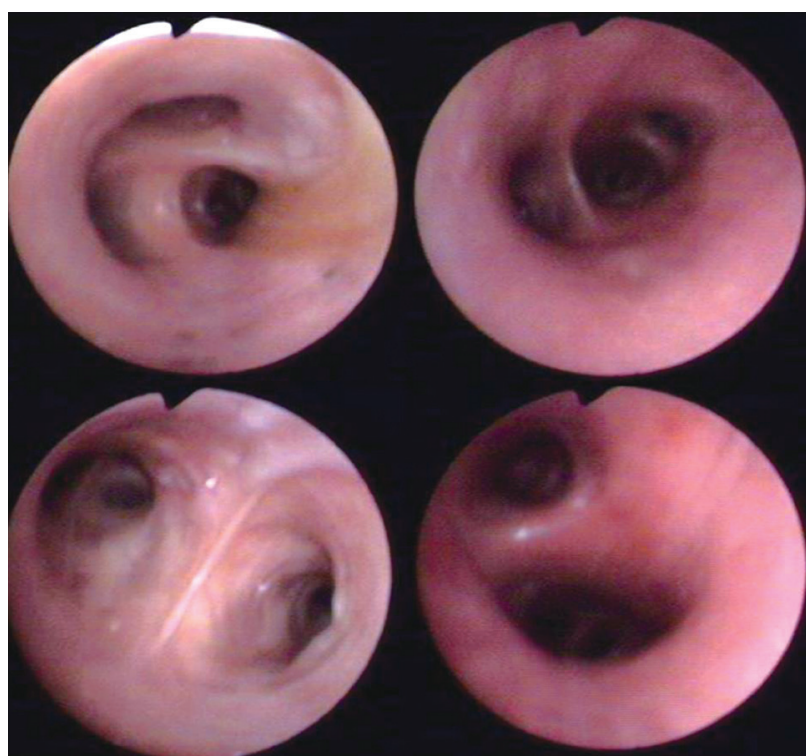

Fig. 4. Bronchoscopy performed on POD 18 showed improved mucosal inflammation and purulent secretion.

lung fields; the $\mathrm{PaO}_{2} / \mathrm{FiO}_{2}$ ratio was decreased at 65 (Fig. 3). Due to the progressive hypoxemic respiratory failure, venous-venous extracorporeal membrane oxygenation was started. XDR A. baumannii with the same resistance pattern was isolated from bacterial cultures of the bronchoscopic washing fluid recovered on POD 7 and sputum on POD 8. At that time, the serum Aspergillus antigen result was negative (0.16) and the cytomegalovirus RQ-PCR result was $<500$ copies $/ \mathrm{mL}$. Because the same XDR A. baumannii was recovered repeatedly from the sputum and bronchial washing fluid and there was no evidence of combined bacterial, mycobacterial, or fungal infection, pneumonia aggravated by XDR $A$. baumannii was strongly suspected. The antibacterial treatment regimen was changed to meropenem (3 $\mathrm{g} /$ day), intravenous (IV) colistin $(4.5 \mathrm{mg} / \mathrm{kg})$, and colistin nebulizer $(4.5 \mathrm{mg} / \mathrm{kg})$ to achieve a synergistic effect on the XDR A. baumannii. The target tacrolimus level was adjusted to 5 to $7 \mu \mathrm{g} / \mathrm{mL}$, and the MMF dose was decreased from 2 to $1 \mathrm{~g} /$ day. With the change in the antibacterial regimen and reduced immunosuppression, the elevated hs-CRP level and leukocytosis regressed on POD $18(7.51 \mathrm{mg} / \mathrm{dL}$ and $10.97 \times 10^{9} / \mathrm{L}$, respectively), and the bilateral lung infiltrations resolved (Fig. 3). Follow-up bronchoscopy on POD 18 showed improved mucosal inflammation and decreased purulent sputum in the allograft lung (Fig. 4). After POD 19, no XDR A. baumannii was cultured in sputum samples retrieved from the tracheostomy site. The combined therapy with meropenem and IV colistin was continued for 2 weeks, with the IV colistin used for 40 days and colistin nebulizer for 86 days in total. Drug-induced acute renal failure developed on POD 16, with a serum creatinine level twice as high as the initial value and decreased urine output. However, the decreased renal function recovered after continuous renal replacement therapy for 23 days. At the time of this writing, the patient was still hospitalized (POD 128) while undergoing a respiratory rehabilitation program to promote generalized buildup.

\section{Discussion}

To date, the impact of colonization of multidrug-resistant organisms in the pretransplant lung has been studied mostly in patients with cystic fibrosis (CF). For example, Pseudomonas aeruginosa colonizes up to $80 \%$ of patients with $\mathrm{CF}$, increasing the risk of infection after transplant by an odds ratio of $4.7 ;[9,10]$ it is also known to be associated with BOS.[11] Burkholderia species colonize $15 \%$ to $22 \%$ of patients with $\mathrm{CF}$, and pretransplant colonization by this organism is associated with increased mortality (hazard ratio, $2.23 ; \mathrm{p}=0.04)$.[12,13] Additional data have been published; international guidelines for the selection of lung transplant candidates, published in 2006,[4] state that pretransplant colonization with multidrug-resistant or panresistant $P$. aeruginosa, methicillin-resistant Staphylococcus aureus, Stenotrophomonas maltophilia, and Aspergillus fumigatus is a relative contraindication to transplantation.

Few papers have examined the impact of $A$. baumannii infection on LT. Most of them reported high short-term mortality due to the severe infection. One case series[6] reported that six patients with transplanted lungs were infected during a hospital outbreak, and four died as a result of this infection with persistent recovery of $A$. baumannii from the respiratory tract despite aggressive treatment. Sopirala et al[14] reported that among the 52 LTs performed in one hospital, 6 patients developed pneumonia in the postoperative period with multidrug-resistant $A$. baumannii, and two died of the infection. Martins et al.[15] described a case of donor transmission of carbapenem-resistant A. baumannii, and the patient died 65 days after transplantation. To our 
knowledge, no previous case reports have described colonization of the pretransplant lung by XDR A. baumannii.

In this case, we continued colistin treatment from the day of the operation because of the infection risk due to colonization. The same organism had been isolated repeatedly from respiratory specimens and caused a severe infection in the early postoperative period. We changed the antibacterial agents after referring to a study reporting the survival benefit of a regimen of carbapenem and colistin (survival, colistin alone vs. carbapenem/colistin: 9\% vs. 60\%, respectively) in solid organ transplant patients infected with XDR A. baumannii .[7] Our patient showed clinical improvement on this combination regimen.

In immunocompromised hosts, $A$. baumannii infections can lead to multiorgan failure and death, but no drug of choice for treatment has been established. Common treatment options are carbapenems, $\beta$-lactamase inhibitors, quinolones, aminoglycosides, minocycline, doxycycline, and polymyxins. New treatment options include minocycline derivatives such as tigecycline, new carbapenems such as doripenem, and new-generation cephalosporins such as ceftobiprole and ceftaroline.[16] MDR A. baumannii is defined as being resistant to at least three antimicrobial classes. For the treatment of MDR A. baumannii, tigecycline has received attention and polymyxin E (colistin) has been widely used. Some recent clinical trials have shown poor clinical outcomes with tigecycline alone,[17,18] and the safety of colistin has been frequently discussed because of its nephrotoxicity. Combination therapy shows synergistic effects when carbapenems, sulbactam, or tigecycline are combined with aminoglycosides, colistin, or rifampin.[19]

Because the major adverse effect of colistin is nephrotoxicity and our patient developed drug-induced renal failure during the hospital course, the continuous use of IV colistin was reconsidered several times. Older studies report an incidence of nephrotoxicity of up to $20 \%$ to $50 \%$, while recent clinical studies have found lower rates of nephrotoxicity of $8 \%$ to $14 \%$, and the serum creatinine levels decreased at the end of the colistin treatment.[20] In this case, the MIC of tigecycline was high ( $\mathrm{MIC}=2$ ), while the colistin, which is less toxic than polymyxin $\mathrm{B}$, had to be administered carefully using strict dose modifications according to the daily laboratory results.

Acinetobacter infection is becoming prevalent as more centers are willing to transplant candidates who are on mechanical ventilation or extracorporeal life support.[8] Colonization of the pretransplant lung by this organism will likely increase with prolonged ventilation, the use of broadspectrum antibiotics, and intensive care unit care. We should also consider the patient's host factors; he was severely immunocompromised after taking immunosuppressants for several years after BMT, which made him more susceptible to bacterial infection. Strategies for dealing with pretransplant bacterial colonization and the choice of post-transplant prophylactic antibacterial agent for Acinetobacter species considering both host factors and the antibacterial spectrum of the organism should be investigated. Furthermore, the impact of the organism on the short-term mortality and long-term morbidity after transplantation, such as BOS, should be analyzed as more case reports are published.

In conclusion, we have reported a case of severe infection by XDR $A$. baumannii that colonized the pretransplant lung. The pretransplantation work-up of patients colonized with $A$. baumannii should consider host immunological factors and the drug resistance pattern of the colonizing A. baumannii.

\section{References}

1) Holm AM, Riise GC, Brinch L, Bjørtuft O, Iversen M, Simonsen S, et al: Lung transplantation for bronchiolitis obliterans after allogeneic hematopoietic stem cell transplantation: unresolved questions. Transplantation 2013; 96: e21-2.

2) Kim YR, Haam SJ, Park YG, Lim BJ, Park YM, Paik $\mathrm{HC}$ : Lung transplantation for bronchiolitis obliterans after allogeneic hematopoietic stem cell transplantation. Yonsei Med J 2012; 53: 1054-7.

3) Soubani AO, Kingah P, Alshabani K, Muma G, Haq A: Lung transplantation following hematopoietic stem cell transplantation: report of two cases and systematic review of literature. Clin Transplant 2014; 28: 776-82.

4) Orens JB, Estenne M, Arcasoy S, Conte JV, Corris P, Egan JJ, et al: International guidelines for the selection of lung transplant candidates: 2006 update--a consensus report from the Pulmonary Scientific Council of the International Society for Heart and Lung Transplantation. J Heart Lung transplant 2006; 25: 745-55. 
5) Fournier PE, Richet H: The epidemiology and control of Acinetobacter baumannii in health care facilities. Clin Infect Dis 2006; 42: 692-9.

6) Nunley DR, Bauldoff GS, Mangino JE, Pope-Harman AL: Mortality associated with Acinetobacter baumannii infections experienced by lung transplant recipients. Lung 2010; 188: 381-5.

7) Shields RK, Kwak EJ, Potoski BA, Doi Y, AdamsHaduch JM, Silviera FP, et al: High mortality rates among solid organ transplant recipients infected with extensively drug-resistant Acinetobacter baumannii: using in vitro antibiotic combination testing to identify the combination of a carbapenem and colistin as an effective treatment regimen. Diagn Microbiol Infect Dis 2011; 70: 246-52.

8) Shoham S, Shah PD: Impact of multidrug-resistant organisms on patients considered for lung transplantation. Infect Dis Clin North Am 2013; 27: 343-58.

9) Bonvillain RW, Valentine VG, Lombard G, LaPlace S, Dhillon G, Wang G: Post-operative infections in cystic fibrosis and non-cystic fibrosis patients after lung transplantation. J Heart Lung transplant 2007; 26: 890-7.

10) Palmer SM, Alexander BD, Sanders LL, Edwards LJ, Reller LB, Davis RD, et al: Significance of blood stream infection after lung transplantation: analysis in 176 consecutive patients. Transplantation 2000; 69: 2360-6.

11) Vos R, Vanaudenaerde BM, Geudens N, Dupont LJ, Van Raemdonck DE, Verleden GM: Pseudomonal airway colonisation: risk factor for bronchiolitis obliterans syndrome after lung transplantation? Eur Respir J 2008; 31: 1037-45.

12) Murray S, Charbeneau J, Marshall BC, LiPuma JJ: Impact of burkholderia infection on lung transplantation in cystic fibrosis. Am J Respir Crit Care Med 2008; 178: 363-71.

13) Kennedy MP, Coakley RD, Donaldson SH, Aris RM, Hohneker K, Wedd JP, et al: Burkholderia gladioli: five year experience in a cystic fibrosis and lung transplantation center. J Cyst Fibros 2007; 6: 267-73.

14) Sopirala MM, Pope-Harman A, Nunley DR, MoffattBruce S, Ross P, Martin SI: Multidrug-resistant Acinetobacter baumannii pneumonia in lung transplant recipients. J Heart Lung Transplant 2008; 27: 804-7.

15) Martins N, Martins IS, de Freitas WV, de Matos JA, Magalhães AC, Girão VB, et al: Severe infection in a lung transplant recipient caused by donor-transmitted carbapenem-resistant Acinetobacter baumannii. Transpl Infect Dis 2012; 14: 316-20.

16) Michalopoulos A, Falagas ME: Treatment of Acinetobacter infections. Expert Opin Pharmacother 2010; 11: 779-88.

17) Petrosillo N, Ioannidou E, Falagas ME: Colistin monotherapy vs. combination therapy: evidence from microbiological, animal and clinical studies. Clin Microbiol Infect 2008; 14: 816-27.

18) Gordon NC, Wareham DW: A review of clinical and microbiological outcomes following treatment of infections involving multidrug-resistant Acinetobacter baumannii with tigecycline. J Antimicrob Chemother 2009; 63: $775-80$.

19) Gallagher JC, Rouse HM: Tigecycline for the treatment of Acinetobacter infections: a case series. Ann Pharmacother 2008; 42: 1188-94.

20) Falagas ME, Kasiakou SK: Toxicity of polymyxins: a systematic review of the evidence from old and recent studies. Crit Care 2006; 10: R27. 